\title{
Correcting etaloning fringes in hyperspectral image cubes of Jupiter using sensor thickness modeling with flat-field data fitting
}

\author{
Erandi Wijerathna, ${ }^{\text {a,* }}$ Emma Dahl, ${ }^{\mathrm{b}}$ David Voelz, ${ }^{\mathrm{a}}$ and Nancy Chanover ${ }^{\mathrm{b}}$ \\ ${ }^{a}$ New Mexico State University, Klipsch School of Electrical and Computer Engineering, \\ Las Cruces, New Mexico, United States \\ ${ }^{b}$ New Mexico State University, Department of Astronomy, Las Cruces, New Mexico, \\ United States
}

\begin{abstract}
Evidence of "fringing" due to optical etaloning was observed in narrowband hyperspectral image cubes of Jupiter collected prior to 2018 at the Apache Point Observatory 3.5-m telescope with the New Mexico State University Acousto-optic Imaging Camera. The etaloning resulted from the use of a back-illuminated, high quantum efficiency CCD. Otherwise successful flat-field correction was ineffective in removing fringes at some wavelengths associated with Jupiter's absorption regions. We describe an etaloning correction method based on a mathematical interference model that assumes a single detection layer. A two-dimensional thickness function for the sensor layer was derived and found to have an overall "dish-shaped" variation along with some finely spaced surface polishing marks. Synthetic fringe frames corresponding to the flat-field and science images were created using the thickness function. Optimized contrast values were found for the synthetic frames and defringed images of Jupiter were generated by separately correcting flat-field and science images using the synthetic fringe frames before applying the final flat-field division. Quantitative analyses of defringed flat-field images showed fringe contrast reductions by factors of 2 to 8 on average and a disk-averaged spectrum of defringed Jupiter data compared favorably with an established spectrum. This defringing approach is applicable to other detectors that can be modeled with a single detection layer and where a sequence of spectral images with adequate wavelength resolution is available. (C) The Authors. Published by SPIE under a Creative Commons Attribution 4.0 Unported License. Distribution or reproduction of this work in whole or in part requires full attribution of the original publication, including its DOI. [DOI: 10.1117/1.JATIS.6.2.028002]
\end{abstract}

Keywords: optical etaloning; fringing; image processing; Jupiter; focal-plane arrays; telescopes.

Paper 19103 received Sep. 26, 2019; accepted for publication Jun. 16, 2020; published online Jun. 27, 2020.

\section{Introduction}

Spurious intensity variations or "fringes" in narrowband astronomical science images can result from the use of components such as backside-illuminated, thinned CCD focal-plane arrays. The fringes generally need to be removed from the science frames before actual analyses can begin. The phenomenon that forms these fringe patterns is an interference effect known as optical etaloning, which occurs when light incident on the detector penetrates the sensor material, reflects off rear surfaces or structures, and interferes with the incident light. This results in fringes that are superimposed on the detected signal. The form of the fringes is primarily dependent on the thickness variations in the sensor layers while the fringe intensities and contrast (visibility) are a function of the wavelength of the incident light. ${ }^{1-5}$ For a thinned, backside-illuminated $\mathrm{CCD}$ with an Si detection layer, the $\mathrm{Si}$ absorption coefficient decreases with increasing wavelength so the detection layer is more easily penetrated at longer wavelengths and internally reflected intensity can be significant enough to produce discernible fringes.${ }^{1,3,4}$ For Si detectors, fringing typically becomes prominent at wavelengths longer than $\sim 700 \mathrm{~nm} .{ }^{1-6}$ Moreover, narrowband light results in higher contrast fringes relative to broadband illumination as there is less spectral averaging of the fringes. ${ }^{3,4}$ We describe and demonstrate an approach for

*Address all correspondence to Erandi Wijerathna, E-mail: erandi@nmsu.edu 
the correction of etaloning effects found in narrowband spectral imagery of Jupiter collected with the New Mexico State University Acousto-optic Imaging Camera (NAIC) at Apache Point Observatory (APO).

Etaloning has been experienced in a variety of astronomical applications, ${ }^{1-4,7-9}$ including the sensors on the Hubble Space Telescope. ${ }^{1,4,7,9}$ It can be mitigated using sensors with thicker detection layers, but the thinned, backside-illuminated devices are attractive because they provide significantly higher quantum efficiencies.

The process of removing fringes after data collection is aptly named "defringing." There are a few defringing approaches discussed in the literature. ${ }^{1,3,6,8}$ Malumuth et al. ${ }^{1}$ suggested a method to develop a thickness function for the detection layer of the CCD using a Fresnel equation model. This method has been used to correct fringes in images from the Hubble Space Telescope. ${ }^{2,4,7}$ Even though these researchers used a limited number of images to support the model, they had prior knowledge of the structure of the CCD, including its layers and nominal thicknesses. Howell ${ }^{3}$ proposed a method to correct fringes of a star field via the use of flat-field frames obtained with illumination from a neon lamp. This was possible in this case because the neon emission line source matched the night-sky emission that produced the fringing. Rojo and Harrington $^{8}$ suggested a method based on wavelet transform and a local spectral technique; however, the method does not completely remove fringes when the fringe contrast is significant. Ren et al. ${ }^{6}$ also suggested a wavelet-associated method to correct fringing in an interference imaging spectrometer. However, these methods are dependent on the application (e.g., observing a planet or a star constellation), prior knowledge of the CCD structure (e.g., constituent layers and nominal thicknesses), and the ability to collect substantial flat-field data.

Correcting the etaloning effects in the narrowband Jupiter images produced by NAIC is important as these data complement infrared and microwave observations of Jupiter's uppermost cloud deck made by the Juno spacecraft, which has been orbiting Jupiter since 2016. Specifically, the processed Jupiter images from NAIC are used to generate reflectance spectra of Jupiter's atmosphere. ${ }^{10}$ Using a radiative transfer code, these visible regime spectra can then be modeled to derive the structure of Jupiter's uppermost cloud deck within the upper troposphere, with depths extending from pressure levels of $\sim 0.001$ to 5 bars. Such measurements are highly complementary to the regions of Juno's instrument sensitivity, which is $\sim 1$ to 10 bars in the infrared regime with the Jovian Infrared Auroral Mapper ${ }^{11}$ and $\sim 1$ to 100 bars in the microwave regime with the microwave radiometer (MWR). ${ }^{12}$ These models of the upper troposphere are used to look for correlations between cloud structure and the distribution of ammonia gas in the deep atmosphere as measured by the MWR, ${ }^{13}$ which can lead to important insights on the nature of motion and weather in Jupiter's deep atmosphere. Furthermore, the hyperspectral image cubes acquired with NAIC are highly complementary to integral field spectra of Jupiter acquired with the Multi-Unit Spectroscopic Explorer (MUSE) instrument on the European Southern Observatory's Very Large Telescope ${ }^{14}$ that are also a part of the ground-based support for the Juno mission. Whereas the MUSE integral field spectra provide full-disk spectra over approximately the same wavelength region as NAIC, the acousto-optic tunable filter (AOTF)-based hyperspectral image cubes result in full-disk images at 241 discrete wavelengths. ${ }^{10}$ Both techniques are powerful tools for probing the tropospheric cloud structure of Jupiter during the Juno era.

\section{NAIC System and Data Collection}

The NAIC instrument, shown in Fig. 1, has been operated with the APO 3.5-m telescope in Sunspot, New Mexico, during the perijove passes of the Juno spacecraft. NAIC utilizes an AOTF operated with a radio frequency (rf) signal where the rf selects the center wavelength of the narrowband filter. Using the NAIC set up, narrowband hyperspectral image cubes of Jupiter are collected from 470 to $950 \mathrm{~nm}$ at 2 -nm intervals. The average spectral resolving power of the filter is $R=205$ (e.g., $\Delta \lambda \approx 3.59 \mathrm{~nm}$ at $\lambda=550 \mathrm{~nm}$ ). For operations prior to 2018 , the focal plane used for NAIC was a $1024 \times 1024$ pixel $^{2}$ (binned $2 \times 2$ to obtain $512 \times 512$ pixel $^{2}$ frames), backsideilluminated, high quantum efficiency CCD that showed evidence of etaloning. Here, we discuss the analysis for the data set that was collected on February 2 and 3, 2017, which coincided with the 4th perijove pass of Juno. In addition, a disk-averaged spectrum result is presented in Sec. 4.4 for data collected on March 26 and 27, 2017, during the 5th perijove pass. 


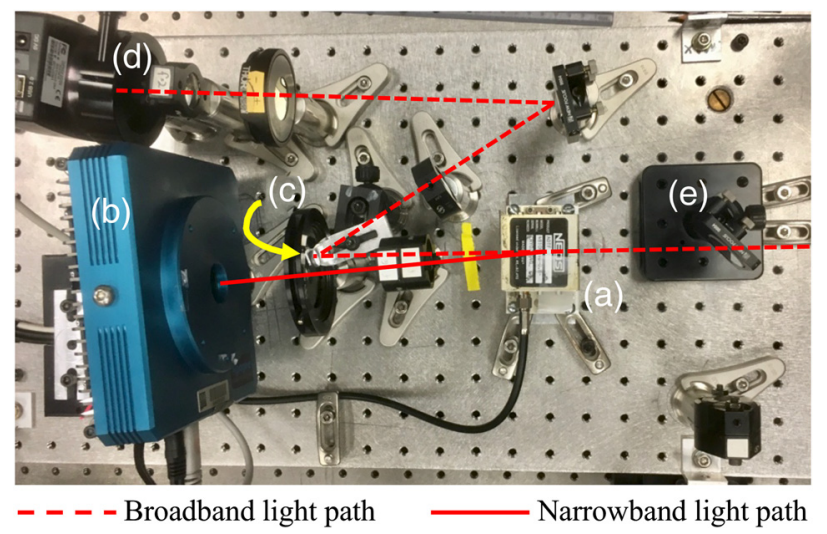

Fig. 1 Optical path diagram of NAIC. The light from the telescope enters (a) AOTF and the narrowband light (solid line) and broadband light (dashed line) are separated in angle. The narrowband light is collected at the (b) CCD and the broadband light is directed to the (d) viewfinder camera using a (c) pickoff mirror. When needed, (e) a turn mirror is used to direct the light beam for laboratory work.

The $512 \times 512$ pixel $^{2}$ science frames collected with NAIC undergo two correction procedures, namely, stray/scattered light correction and flat-fielding correction. As shown in Fig. 1, light from the telescope enters the AOTF from the right, and upon exiting the filter, the narrowband signal light angularly separates from the broadband light. The narrowband light is sent to the CCD to form the science image while the broadband light is redirected with a pickoff mirror. However, some stray broadband light is scattered into the narrowband path and this stray light component can be observed separately at the focal plane by switching off the rf drive signal to the AOTF. Thus, to perform the stray/scattered light correction, the broadband signal in the "rf-on" narrowband image of the planet is removed by subtracting a corresponding "rf-off" image. This procedure is analogous to a conventional dark and bias frame correction. The second correction, the flat-fielding correction, is performed to remove spatial (pixel-to-pixel) variations in the instrument's response. Quartz-tungsten-halogen (QTH) lamps within the closed dome are used to illuminate the $3.5-\mathrm{m}$ telescope mirror covers, producing uniform illumination at the focal plane that can be imaged to generate flat-field images. Similar to science frames, flat-field images are collected at 2-nm intervals and corresponding rf-off frames are subtracted. The science frames are then divided by the corresponding flat-field frames (appropriately normalized) to complete the flat-fielding correction.

For the NAIC instrument prior to 2018, etaloning fringes were visible in the two-dimensional (2-D) flat-field images for wavelengths longer than $\sim 750 \mathrm{~nm}$. Figure 2(a) shows count rate (intensity) as a function of wavelength for the center pixel in a flat-field cube. The periodic signature of etaloning is evident for wavelengths $>750 \mathrm{~nm}$ where the signature is superimposed on large slope-like features peaking around $820 \mathrm{~nm}$ that are due to a combination of the AOTF transmission response and the sensor quantum efficiency response. The linearly sloped region where the wavelength ranges from 820 to $940 \mathrm{~nm}$ [shaded in Fig. 2(a)] is the portion of the flat-field data that was used for calculation of the sensor thickness function (Sec. 3.1). The error estimates associated with the intensity measurements for the pixel shown were found by computing the standard deviation over three available data sets and also averaging with the error results for the eight neighboring pixels to improve the statistical accuracy. The slope of the intensity was removed before performing the standard deviation calculation and the result for the central pixel $(256,256)$ is shown in Fig. 2(b). The error is $<2 \%$ of the mean count rate and is consistent with the expected Poisson noise, which is between $\sim 2.4 \%$ and $1 \%$ of the mean pixel count.

Figures 3(a) and 3(b) show examples of a flat-field frame and a Jupiter image with etaloning effects at a wavelength of $926 \mathrm{~nm}$. At shorter wavelengths $(<\sim 800 \mathrm{~nm})$, fringes are not readily visible by eye in Jupiter science images because the fringe contrast is low and the banding of Jupiter's atmosphere can act as camouflage. However, in general, as the contrast of the fringes becomes larger at longer wavelengths, fringes become evident even in the Jupiter science 

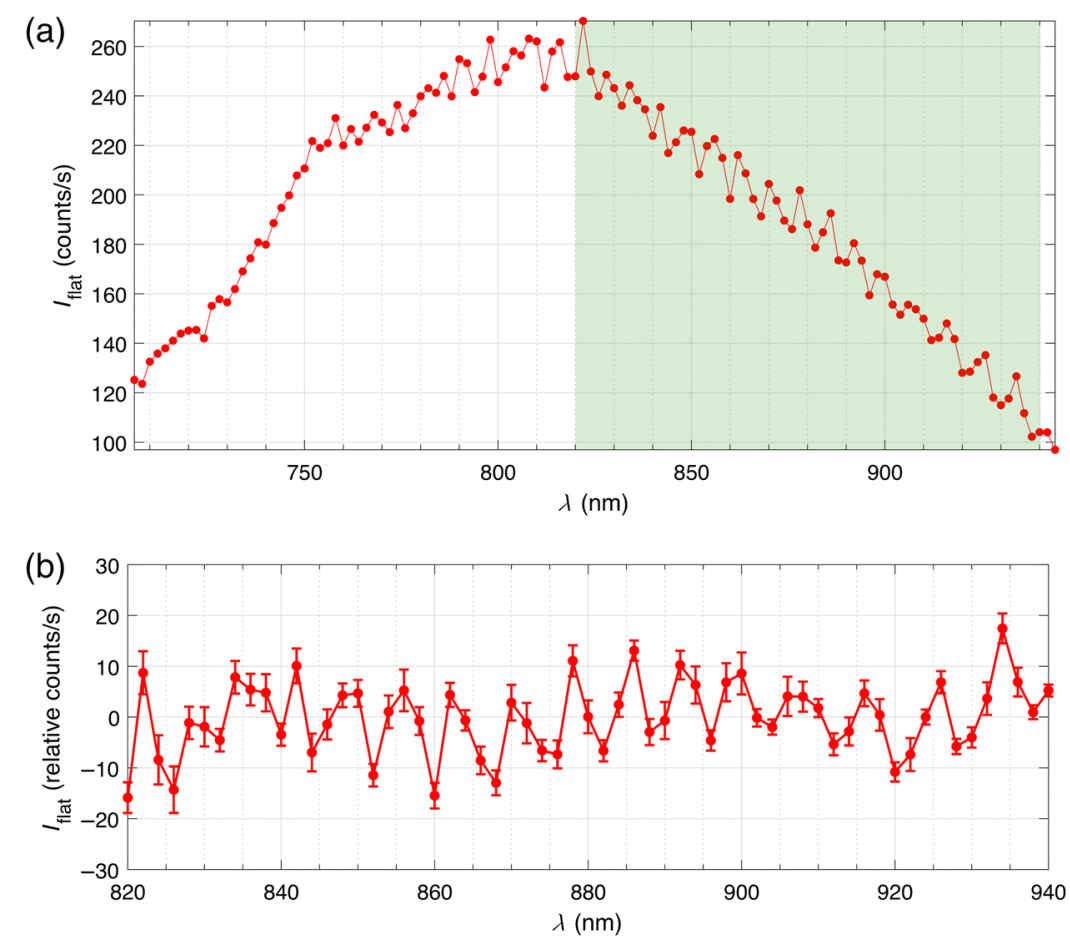

Fig. 2 At pixel $(x, y)=(256,256)$, (a) flat-field intensity as a function of wavelength, where the linearly sloped intensity region (shaded in green) is used for the thickness calculation (see Sec. 3.1) and (b) slope-removed flat-field intensity of the shaded region with error bars. The periodic variations due to etaloning are clearly resolved relative to the error amplitudes.

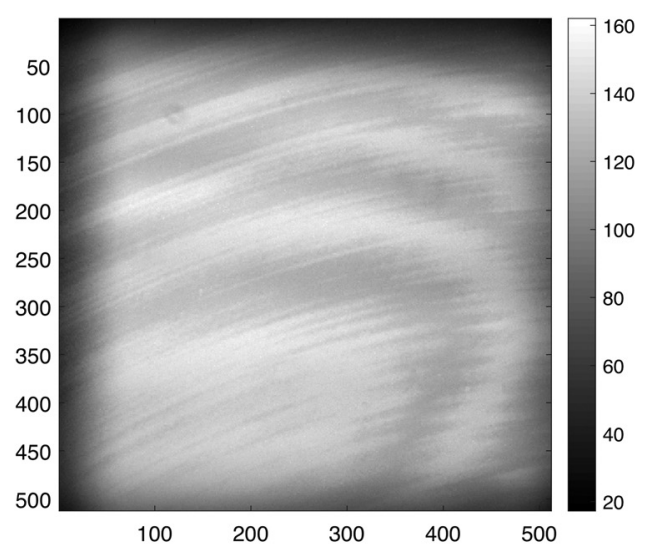

(a)

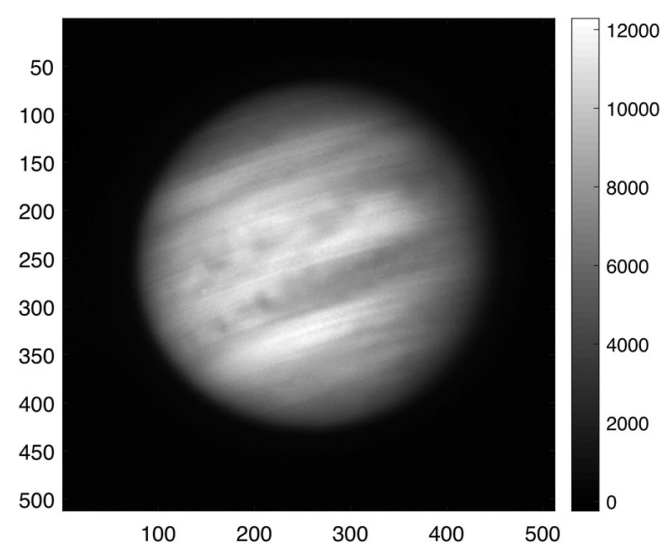

(b)

Fig. 3 (a) Flat-field and (b) Jupiter images with etaloning fringes at $\lambda=926 \mathrm{~nm}$. Thin fringes near the bright areas are most noticeable in (b).

images. Several intensity features, particularly identifiable in the flat-field frame [Fig. 3(a)], suggest certain characteristics of the detector surface and internal surfaces or layers. Large fringes, roughly curling around an area near the lower-left corner of the frame, are due to a "dish-shaped" thickness function, where thickness is defined by the distance from the sensor face to an internal surface that allows reflection. Additional thin fringe-like features appear to be caused by polishing grooves on the top face of the silicon. Other features in Fig. 3(a) such as loss of sensitivity at the edges and some horizontal banding are due to the transmission characteristics of the AOTF. A notional sketch of a portion of the detector cross section is shown in Fig. 4. 


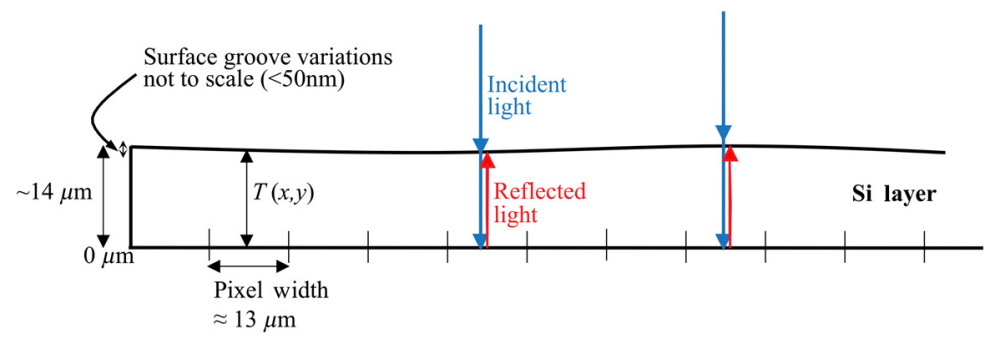

Fig. 4 A notional profile illustration of a portion of the silicon sensor with a ray diagram of incident and reflected rays for two pixels. $T(x, y)$ is the distance from the sensor face to an internal surface at pixel $(x, y)$. The thickness associated with a pixel is considered to be constant and the period of the small polishing grooves on the surface extends more than 5 pixels. The thickness also contains a dish-shaped component that spans the full sensor area.

It is worth noting that a typical sensor contains multiple internal surfaces and associated layers other than the detection layer, such as antireflective coatings, insulation layers, and substrate layers. ${ }^{1,2}$ Therefore, reflections from these other features contribute to further fringing. We found that the single-layer model (sensor face and one internal surface) was generally effective for describing the primary etaloning effects that are seen in our data. However, it is likely that unaccounted surfaces and layers create some effects in our results as described in Sec. 4.3.

Since etaloning produces fringing in both flat-field images and Jupiter images, it can be anticipated that the fringes in the science images can be corrected by flat-field division. However, this is only true if the fringing pattern in both images is the same. Several conditions may invalidate this assumption, for example, if the optical spectral energy distribution of the science source (e.g., Jupiter) is not the same as the spectral content of the flat-field source (e.g., QTH lamps) within the filter bandwidth, or if artifacts in the images such as noise influence the fringe contrasts differently. As demonstrated in Sec. 4.3, for much of the wavelength range, a typical flat-field correction can successfully remove the fringing from the science images. However, for some absorption features, especially in Jupiter's prominent methane $\left(\mathrm{CH}_{4}\right)$ band at $\sim 890 \mathrm{~nm}$, fringing is unintentionally enhanced by the usual flat-fielding process. It is these residual and enhanced fringing scenarios that we target in our correction approach.

The exact physical attributes of our commercial sensor are not available. However, because the NAIC data set provides a sequence of images at small wavelength intervals, it is possible to deduce the thickness function from the flat-fields image spectral data. Therefore, to accurately remove the fringing patterns from the flat-field and science images, we formulate an interference model based on wave optics field equations. Comparing the model with a sequence of flat-field images as a function of wavelength, we develop the 2-D physical thickness function of the CCD at each pixel. Based on the derived thickness function, we build synthetic fringe model frames as a function of wavelength to correct fringes in the flat-field and Jupiter images. The method is entirely computational and no prior knowledge of the sensor schematics or further data collection is necessary.

In previous work, ${ }^{15}$ we presented the preliminary algorithms and results of this defringing method. Even though the final corrected science images showed a promising level of fringe removal, the computed thickness function presented nonphysical jumps due to an ambiguity that can arise in the thickness computation. In this work, we have refined our algorithms to manage the ambiguity and included a wavelength-dependent refractive index in the computations. Furthermore, we present more detailed analyses of the fringe effects in our image cube data and a quantitative assessment of the contrast reduction provided by our defringing method.

\section{Approach}

Assuming uniform illumination, normal incidence, and a sensor consisting of a single layer (see Fig. 4), the normalized complex field $U(x, y ; \lambda)$ that is found just within the sensor face can be given by 


$$
U(x, y ; \lambda)=1+\alpha(\lambda) \exp \left[-j \frac{2 \pi}{\lambda} n(\lambda) 2 T(x, y)\right]
$$

where $(x, y)$ is a pixel location on the sensor face, $\lambda$ is the central wavelength of the filter bandpass, $j=\sqrt{-1}, T(x, y)$ is the sensor layer thickness function, $2 \pi / \lambda$ is the wavenumber, and $n(\lambda)$ is the real part of the refractive index of the sensor material (silicon in our case). The unit value represents the normalized amplitude of an incident optical field that just enters the detection layer and the second term describes a field component that travels through the sensor layer to the reflecting surface and back (see Fig. 4). The parameter $\alpha(\lambda)$ accounts for the ability of the reflected field to interfere with the incident field. The phase in the complex exponential represents the optical path delay due to the down-and-back propagation in the layer. Multiplying $T(x, y)$ by two accounts for the round-trip distance. The intensity $I(x, y ; \lambda)$ from the summation (superposition) of the two coherent field components is calculated as

$$
\begin{aligned}
I(x, y ; \lambda) & =|U(x, y ; \lambda)|^{2}=1+\alpha(\lambda)^{2}+2 \alpha(\lambda) \cos \left[\frac{2 \pi}{\lambda} n(\lambda) 2 T(x, y)\right] \\
& \approx 1+2 \alpha(\lambda) \cos \left[\frac{2 \pi}{\lambda} n(\lambda) 2 T(x, y)\right],
\end{aligned}
$$

where typically $\alpha(\lambda)^{2} \ll 1$. Equation (2) is our analytical model for the etaloning process and describes a normalized intensity with a cosine term that defines the fringes resulting from etaloning.

The quantity $2 \alpha(\lambda)$ is the wavelength-dependent contrast for the etaloning fringes. The parameter $\alpha(\lambda)$ thus embodies the various physical processes that affect the fringe amplitude including: (1) the relative intensities of the incident and reflected waves, (2) the coherence of the incident light, which is related to the spectral energy distribution of the source and the bandwidth of the observing filter, and (3) measurement noise that can obscure the fringes. The model does not distinguish between these processes but assumes that $\alpha(\lambda)$ represents their combined effect.

Our defringing process requires a data-driven empirical derivation of both $T(x, y)$ and $\alpha(\lambda)$. In overview, Eq. (2) is applied in an initial search process to recover the thickness function $T(x, y)$ from flat-field data. It is worth noting that $T(x, y)$ may not represent the exact thickness of the sensor layer as the detection process can occur some distance within the layer, but this issue is not critical to the modeling. The recovered thickness function is inserted back into Eq. (2) to create normalized synthetic intensity frames as a function of wavelength with fringe features that correspond to the etaloning fringes. The actual correction of an image at a particular wavelength involves division by the appropriate synthetic intensity frame with a value of $\alpha(\lambda)$ that is found with a search procedure that minimizes the fringe artifacts in the image. As seen in Sec. 3.2, an important feature of our approach is that the flat-field and science frames are initially corrected separately before the science frames are finally flat-fielded.

\subsection{Deriving Thickness Function}

The first step in removing the fringes is to determine the effective thickness function $T(x, y)$ of the CCD sensor layer. Our approach was to apply a search procedure at each pixel to fit Eq. (2) to the flat-field intensity data. For each pixel, the intensity was extracted from the flat-field frames for the wavelength range from 820 to $940 \mathrm{~nm}$ (61 values), which corresponds to an approximately linearly sloped response region (Fig. 2). The data for each pixel were then processed using a high-pass filter to remove the slope from the flat-field intensity profile as shown in Fig. 2(b). It is worthy to note that because the thickness function is an argument in the cosine in Eq. (2), it is primarily the wavelength-dependent frequency and phase of the sinusoid signature in Fig. 2(b) that need to be fitted with Eq. (2) to find $T(x, y)$.

To apply Eq. (2) to a pixel at $(x, y)$, the term $\alpha(\lambda)$ was replaced with a constant value $\alpha_{\mathrm{rms}}(x, y)$, which is the root mean square (rms) value of the slope-removed flat-field signal for the particular pixel. For $n(\lambda)$, we used silicon refractive index values tabulated by Green ${ }^{16}$ for 


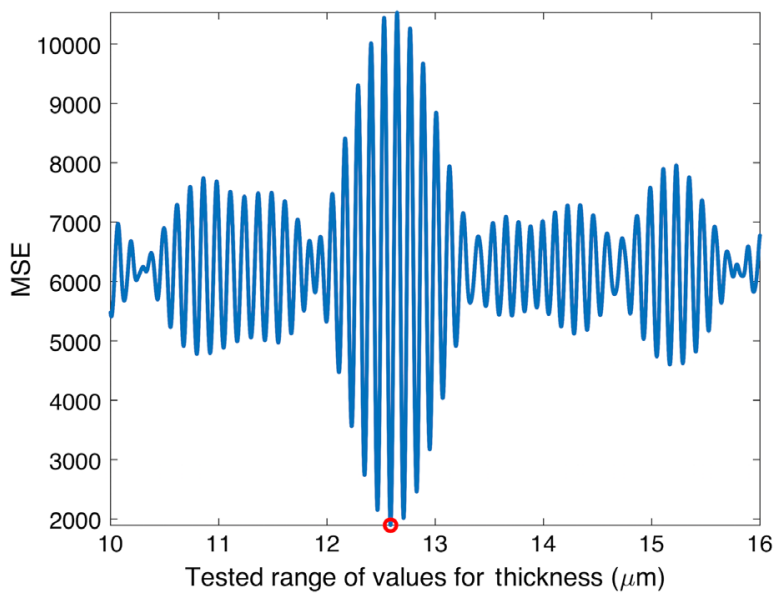

Fig. 5 Behavior of the MSE between the modeled intensity and the slope-removed flat-field intensity for a range of thickness values at pixel $(x, y)=(256,256)$. The red circle at $12.6 \mu \mathrm{m}$ indicates the thickness value corresponding to the minimum MSE.

every $10 \mathrm{~nm}$ in the wavelength range of interest at room temperature $(300 \mathrm{~K})$. Linear interpolation was applied to find the refractive indices corresponding to the 61 wavelengths of interest.

Next, a set of test thickness values within a selected range was applied to Eq. (2). For each test value, the mean square error (MSE) between the computed intensity and the slope-removed flatfield intensity was calculated. The test value that produced the minimum MSE was chosen to be the thickness at the considered pixel. The example plot in Fig. 5 of the MSE versus thickness values for the pixel at $(256,256)$ shows that the MSE behaves in an oscillatory manner with the minimum near $12.6 \mu \mathrm{m}$. The oscillation period is proportional to the finite wavelength range (820 to $940 \mathrm{~nm}$ ) that is available from the flat-field data. Figure 6 shows a comparison of the slope-removed flat-field intensity $\left(I_{\text {flat }}\right)$ and the intensity result from Eq. (2) for the thickness value obtained through the MSE search $\left(I_{\text {model }}\right)$. Although the modeled intensity cannot account for all the detailed amplitude changes in the data, the model shows good agreement in terms of the frequency and phase, which is most relevant to the thickness fit.

It is worth noting that our choice of $\alpha_{\mathrm{rms}}(x, y)$ is not critical to the thickness results because the minimum MSE is primarily a function of the frequency and phase of the model signal rather than the amplitude. Other choices for $\alpha(x, y)$ produced the same results, but $\alpha_{\text {rms }}(x, y)$ is convenient and provides a similar amplitude in the model result as in the flat-field data. With regard to variations in $n(\lambda)$, an Si detection layer in a CCD may contain dopants and other impurities, ${ }^{1,2}$ and the refractive indices for $\mathrm{Si}$ presented in the literature may also lack accuracy. ${ }^{1,16}$ Nevertheless, these differences are typically $<0.5 \% 1,16$ and we found from simulation results that such variations can create up to $20-\mathrm{nm}$ differences in the recovered thickness function but do not change the physical form of the function nor the level of final correction in the images.

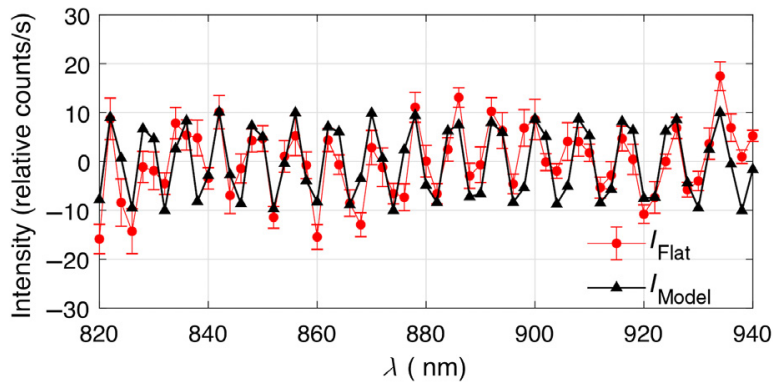

Fig. 6 The slope-removed flat-field intensity $\left(I_{\text {flat }}\right)$ and the modeled intensity $\left(I_{\text {model }}\right)$ at pixel $(x, y)=$ $(256,256)$ for the thickness value shown in Fig. 5 . Only the match between fringing frequency and phase (not amplitude) is significant for determining the layer thickness. 
The search approach described above was repeated for all pixels to obtain the 2-D thickness function $T(x, y)$. However, an issue with this approach is that the oscillatory nature of the MSE result (Fig. 5) combined with noise in the data can cause the minimum MSE metric to incidentally "jump" between two adjacent valleys. This leads to some ambiguous thickness jumps between pixels in the 2-D thickness profile, as noted in our previous work. ${ }^{15}$ Here, we assumed that large, discrete thickness jumps between adjacent pixels are extremely unlikely and developed an algorithm to exclude these features. We first computed the thickness for a starting pixel at the center of the frame and then limited the allowable change when computing the thickness for adjacent pixels. The thickness computation was typically started at the center pixel $[(x, y)=$ $(256,256)]$ because the signal intensity is relatively less noisy due to better AOTF sensitivity at the center. For this starting pixel, the search was conducted over a thickness range from 10 to $16 \mu \mathrm{m}$ in $0.01-\mathrm{nm}$ intervals. We investigated larger thickness ranges, for example 5 to $30 \mu \mathrm{m}$, and found the global minimum does not change. The thickness search for each pixel is then stepped out toward the edges of the sensor. The allowable change for the thickness search in subsequent pixels was limited to $\pm 60 \mathrm{~nm}$ of the average thickness of the available adjacent pixels. The $\pm 60 \mathrm{~nm}$ change was chosen because the typical spurious amplitude step of the profile was $\sim 120 \mathrm{~nm}$. The algorithm was also tested with different starting pixels and we found the relative thickness profile was essentially unchanged, although, depending on the choice of the starting pixel, the mean thickness of the 2-D profile could change by $\pm 120 \mathrm{~nm}$. However, this difference has no effect on the final defringing results.

\subsection{Correcting Etaloning Effects}

The next step for etaloning correction involves separately removing the fringes from each science frame and flat-field frame. Correcting the Jupiter and flat-field images separately compensates for the issue that the frames may have been formed with differing optical spectral content or other differences such as noise levels that affect the fringe contrast. Fringing is removed by dividing the frame of interest by a normalized synthetic fringe model frame ("fringe frame") that is created by applying the derived 2-D thickness function along with an appropriate value of $\alpha(\lambda)$ in Eq. (2). It is not feasible with our data to recover $\alpha$-values for each pixel in a single spectral frame. Rather, a single value for $\alpha(\lambda)$ is found for each frame through an iterative process of minimizing the overall presence of the fringes in the frame.

Our metric for determining the amount of fringing present in a given frame is to compute the 2-D spatial power spectrum of the frame and examine a region of the power spectrum that contains the fringing signature. Figure 7(a) shows a grayscale representation of the power spectrum for a flat-field image where the tilted "bow-tie" feature is a signature of the fringing formed by our particular CCD. Normalized synthetic fringe frames for a range of $\alpha$-values were created with the 2-D thickness function applied to Eq. (2). The flat-field frame was divided by each synthetic frame trial and the resulting power spectra were monitored. The $\alpha$-value for the best

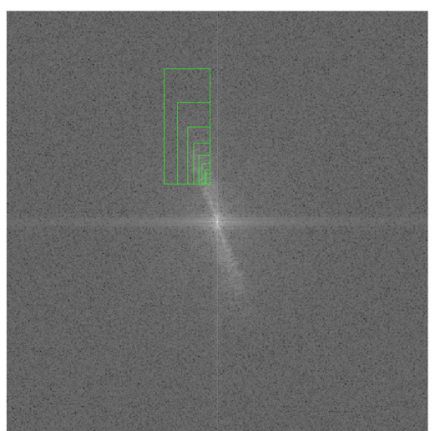

(a)

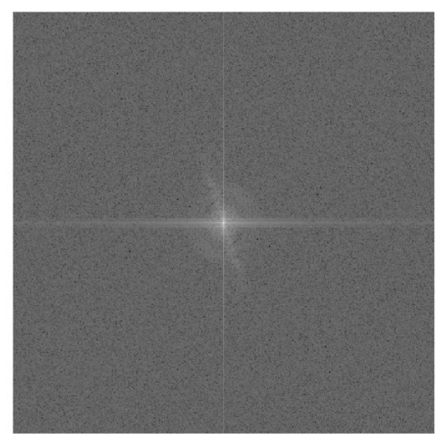

(b)

Fig. 7 Power spectrum of a flat-field at $\lambda=848 \mathrm{~nm}$ (a) before and (b) after defringing (i.e., divided by the synthetic fringe frame). Figures are shown in log scale for display purposes. The green boxes in (a) are example regions that are examined to minimize the fringe signature. 
correction was found by minimizing the integrated power within an appropriate spectral region. Multiple regions within the power spectrum were examined for each frame and the bestcorrection $\alpha$-values were averaged to reduce the error dependence on the region chosen. The standard deviation of the results for the multiple regions was also calculated to determine the sensitivity of the method to the region selection. An example set of regions for the analysis is shown by the green boxes in Fig. 7(a) where the areas were stepped by a factor of 2 while keeping the aspect ratio the same. Only boxes in one quadrant are used as the power spectrum of a real-valued input is symmetric about dc.

At every wavelength and for a considered region, the search for $\alpha$ was initially conducted in the range from -0.03 to 0.04 at intervals of $10^{-3}$. Once an initial solution was found in this range, another iteration of the search was conducted at \pm 0.002 around the initial solution in intervals of $10^{-6}$ to improve the accuracy of the solution. Although the contrast $(2 \alpha)$ is typically $<0.05(5 \%)$, the initial range for the $\alpha$ search was chosen to be somewhat larger to account for outlying values. The search range included negative values because the best-correction result could be slightly negative due to a response aspect of the model that is discussed in Sec. 4.3. The two-step search for $\alpha$-values made the search computationally efficient in terms of time and memory. Figure 7(b) shows the power spectrum result for the example flat-field frame after dividing by the synthetic fringe frame with the best-correction $\alpha$-value. This correction procedure was applied individually to all flat-field frames over the full wavelength range to get the best-correction $\alpha$-value $\left(\alpha_{\text {flat }}\right)$ as a function of wavelength.

Next, the same steps were repeated for the Jupiter images to determine the best-correction $\alpha$-values $\left(\alpha_{\text {jupiter }}\right)$ for the synthetic fringe frames as a function of wavelength, although more care needed to be taken when examining the power spectrum to avoid components associated with Jupiter's structure and banding. A fringe-corrected Jupiter image was obtained by dividing by the appropriate synthetic fringe frame. The final science image was the fringe-corrected Jupiter image divided by the associated fringe-corrected flat-field image that is normalized by the respective mean.

\section{Results}

\subsection{Thickness Function}

Figure 8 shows the three-dimensional (3-D) projection and the 2-D profile of the thickness function obtained with our approach. The dish-shaped thickness between the sensor face and the internal layer is apparent in the 3-D projection of the thickness function and the 2-D profile clearly shows indications of polishing grooves on the top face that are consistent with the fringe characteristics seen in Fig. 3(a). Furthermore, the 3-D projection of the thickness function

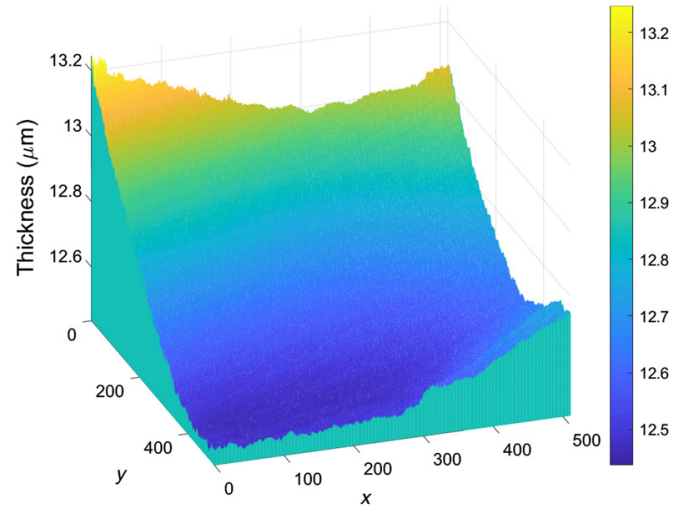

(a)

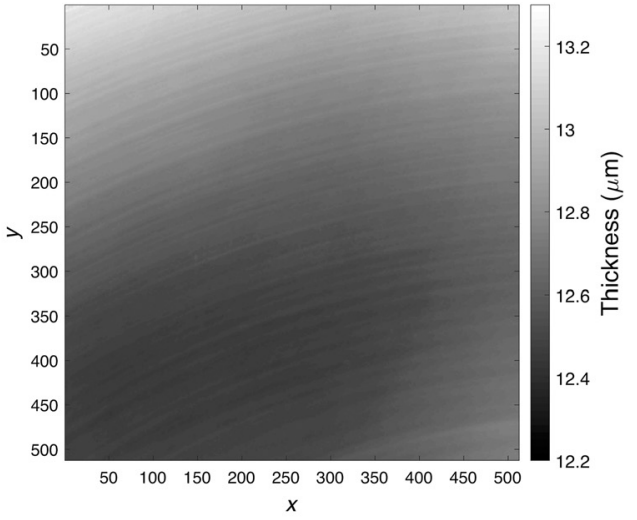

(b)

Fig. 8 Thickness function $T(x, y)$ obtained by minimizing the MSE (a) 3-D projection and (b) 2-D profile in grayscale. 
Wijerathna et al.: Correcting etaloning fringes in hyperspectral image cubes of Jupiter...
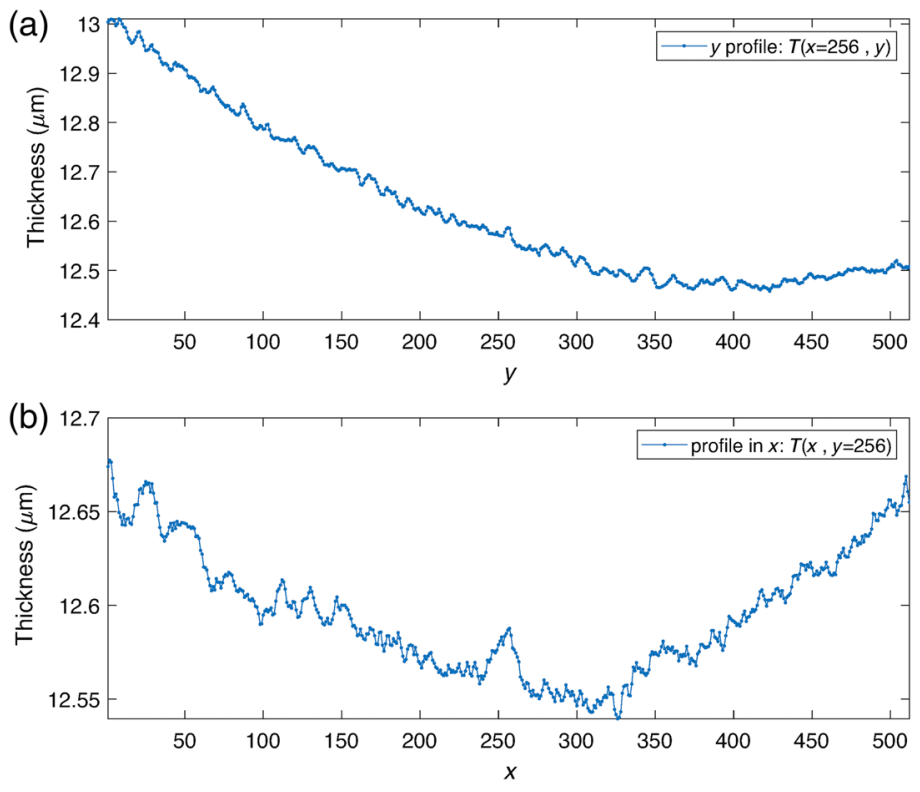

Fig. 9 The 1-D cross-section profiles of the thickness function $T(x, y)$ at (a) $x=256$ and (b) $y=256$.

indicates different curvatures in $x$ and $y$ directions. These thickness features are also visible in one-dimensional (1-D) profiles of the thickness function in the $x$ and $y$ directions along the center of the CCD (Fig. 9). Notably, the 1-D profiles show no evidence of the discrete thickness jumps between adjacent pixels that can arise from noise affecting the MSE search.

As is evident from the thickness function figures, the mean thickness is about $12.67 \mu \mathrm{m}$. However, depending on the starting pixel point for the thickness search and the noise present in the collected data, an uncertainty of $\sim \pm 0.120 \mu \mathrm{m}$ can be expected for the computed thickness.

\subsection{Corrected Images}

Figure 10 shows an example of a synthetic fringe frame obtained with the derived thickness function inserted in Eq. (2). The synthetic fringes are comparable to the fringes apparent in the flat-field frame of Fig. 3(a).

Examples of fringe-corrected flat-field images for four different wavelengths are shown in Fig. 11. These examples represent the onset of fringes $(726 \mathrm{~nm})$, before and within one of the

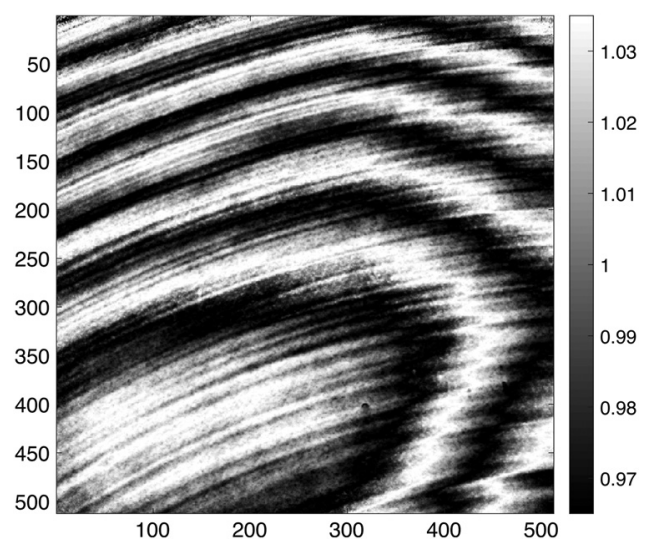

Fig. 10 Synthetic fringe pattern derived with thickness function at $\lambda=848 \mathrm{~nm}$ and best-correction $\alpha=0.0175$ [see Eqs. (1) and (2)]. 


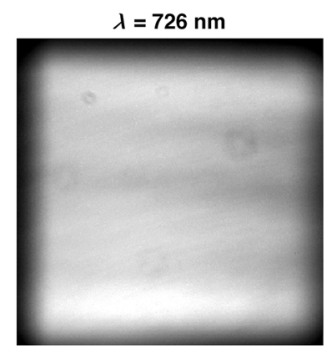

(a)

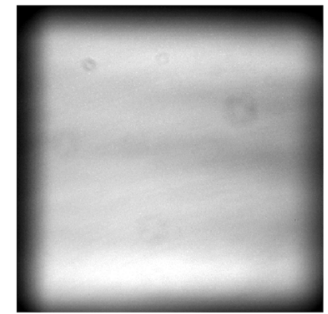

(e)

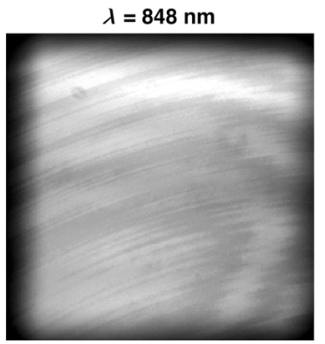

(b)

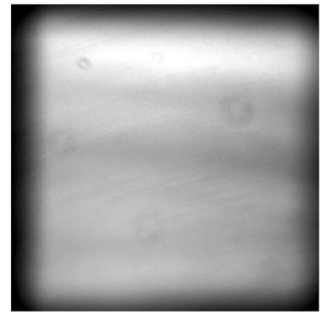

(f)

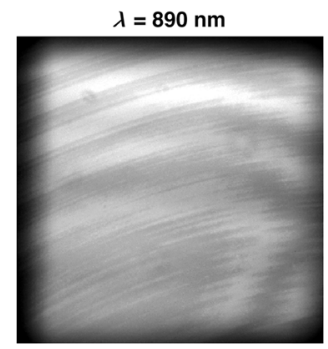

(c)

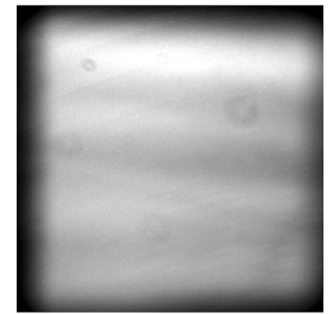

(g)

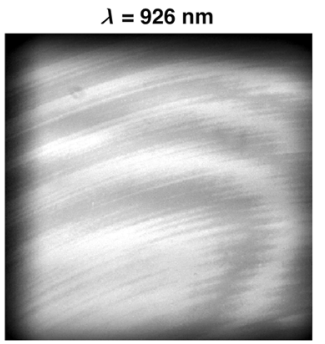

(d)

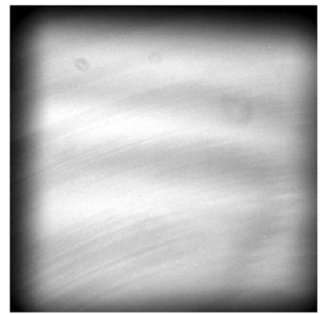

(h)

Fig. 11 Flat-field image correction examples, left to right in each row: $\lambda=726,848,890$, and $926 \mathrm{~nm}$. (a)-(d) Original flat-field images and (e)-(h) defringed flat-field images. Image contrast is enhanced to emphasize features.

$\mathrm{CH}_{4}$ absorption regions of Jupiter (848 and $890 \mathrm{~nm}$ ), and near the end of our data collection range $(926 \mathrm{~nm})$. The top row shows the frames with no correction and illustrates that the fringe contribution is minimal for $726 \mathrm{~nm}$ but becomes more pronounced as the wavelength increases. The bottom row shows the frames after correction and demonstrates the effectiveness of our fringe correction method. A few residual fringe contributions are apparent in the corrected and 926-nm frames. The horizontal banding and the spot-like artifact in the corrected frames are due to the AOTF transmission response and a contaminant on an optical surface. The contrast of these response-related artifacts can be as high as 30\%. These features are corrected in the Jupiter images with the final division by the flat-field frames.

Figure 12 shows example results for the Jupiter images. The top row shows the final science frames where fringe and flat-field corrections have been completed. Even in uncorrected Jupiter frames, it can be difficult to visually identify fringing and transmission response artifacts because of the banding on the planet. Therefore, in the bottom row of Fig. 12, we show the percentage difference between the final science frames and the original uncorrected Jupiter frames. These difference maps illustrate the combination of AOTF/sensor transmission response and etaloning artifacts that are removed from the Jupiter images. The results illustrate that the response corrections tend to dominate but fringe structures are visible in the 848- and 926-nm difference frames. The lack of fringes in the $890-\mathrm{nm}$ difference frame is discussed in Sec. 4.3.

By direct examination of several positions on the flat-field images, the contrast values $(2 \alpha)$ due to the fringes were measured. In the flat-field images before correction, the peak fringe contrast varies between $\sim 5 \%$ and $\sim 2 \%$ (i.e., 0.05 and 0.02 ) depending on the area of the sensor and the wavelength. However, at longer wavelengths $(>890 \mathrm{~nm})$, the overall fringe contrast in the uncorrected flat-fields tends to be close to or above $\sim 5 \%$. After correction, the fringe contrast on average decreases to $\sim 2 \%$ to $1 \%$. This shows that our defringing method reduced the etaloning fringes by at least a factor of $\sim 2$; however, depending on position/wavelength, the correction can be as much as a factor of $\sim 8$.

\subsection{Best-Correction $\alpha$-Values Discussion}

Plots of the best-correction $\alpha$-values for the flats $\left(\alpha_{\text {flat }}\right)$ and Jupiter $\left(\alpha_{\text {Jupiter }}\right)$ as a function of wavelength (Fig. 13) display features that allow further interpretations related to defringing correction, model behavior, and data signal levels. For $\lambda<700 \mathrm{~nm}$, there are no observable fringes in the flat-field or Jupiter images; therefore, the $\alpha$-values for both are near zero. 


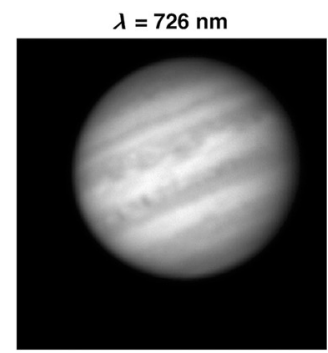

(a)

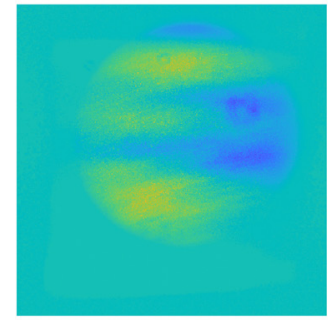

(e)

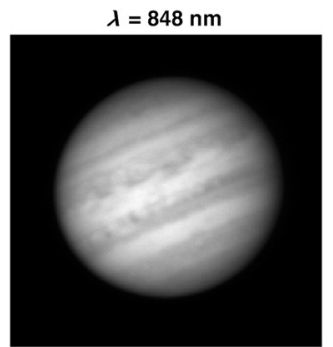

(b)

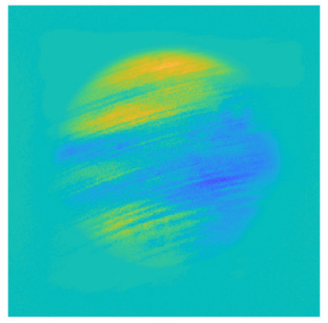

(f)

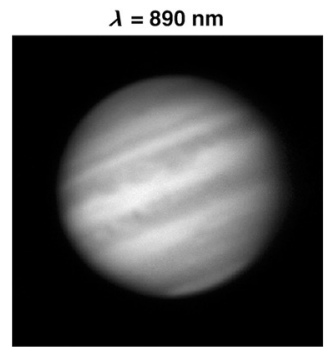

(c)

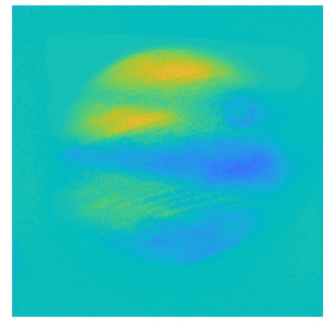

(g)

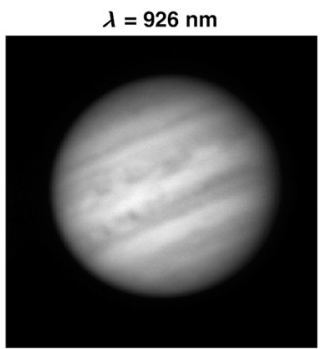

(d)

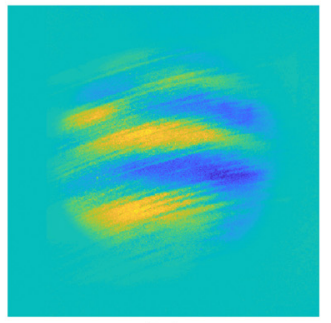

(h)

Fig. 12 Jupiter image correction examples, left to right in each row: $\lambda=726,848,890$, and $926 \mathrm{~nm}$. (a)-(d) Jupiter images corrected using synthetic fringe frames and divided by the defringed flat-fields. Image contrast is enhanced to emphasize features. (e)-(h) Percentage difference per pixel between original Jupiter images and the final corrected Jupiter images. The blue color intensity indicates negative differences $(0 \%$ to $-35 \%)$ and yellow color intensity indicates positive difference $(0 \%$ to $+35 \%)$.

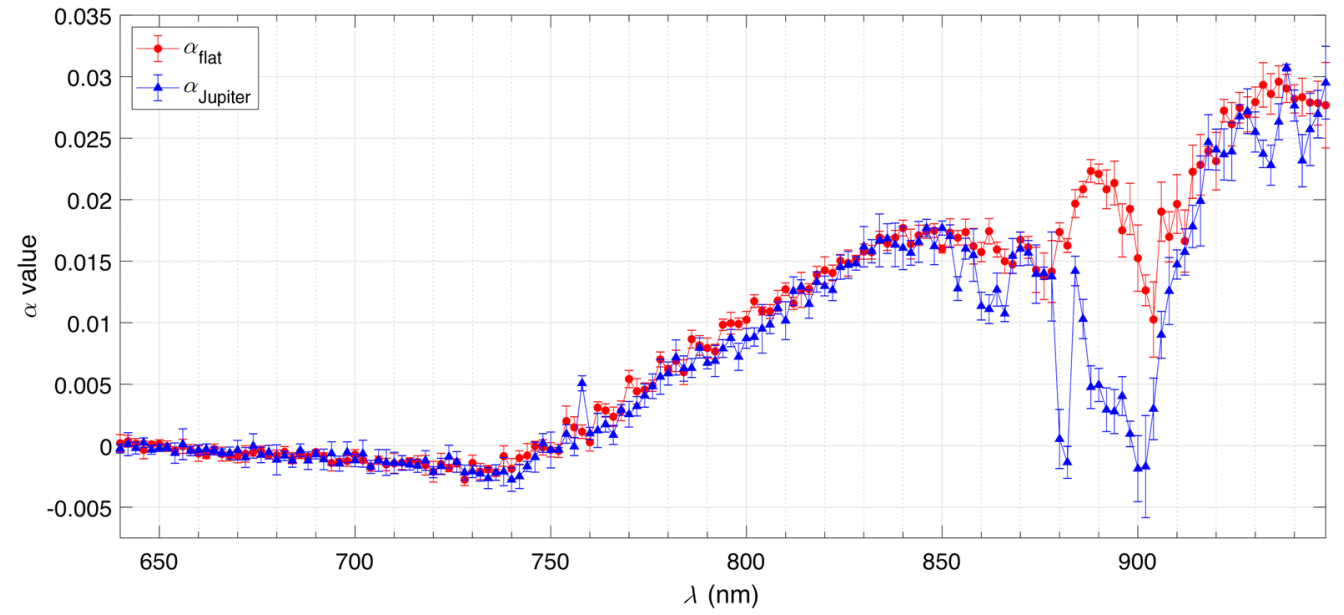

Fig. 13 Best-correction $\alpha$-values [see Eqs. (1) and (2)] as a function of wavelength for the defringing results. The error bars indicate the sensitivity of the power spectrum search method as described in Sec. 3.2.

As the wavelength increases $(\lambda>740 \mathrm{~nm})$, fringes appear as indicated by the increasing $\alpha$-values. The $\alpha$-values continue to rise with wavelength because the $\mathrm{Si}$ absorption decreases with wavelength providing more internally reflected intensity. When $\alpha_{\text {flat }}$ and $\alpha_{\text {Jupiter }}$ have comparable values for a particular wavelength, it indicates that the fringe patterns in both the flat-field and Jupiter frames have similar contrast levels and the correction of the science frames could likely be done with a conventional flat-field division.

A curious feature in Fig. 13 is the slope and negative values for both curves in the 650- to 740-nm range. This corresponds to a contrast-reversed fringe correction. Through numerical simulation studies, we found that an $\alpha$-value oscillating with wavelength can arise if the single-layer model [Eqs. (1) and (2)] is applied to a volume with multiple internal reflecting 
surfaces. Specifically, if a second weak reflecting surface is near the principal reflecting surface, then a portion of a slowly oscillating response can appear like the negative feature in Fig. 13. In effect, this feature represents the response of our model to multiple layers. We believe this is the explanation for this feature and our simulation results show that the single-layer model with this response is still effective in the defringing process for multilayer situations where one surface reflection is dominant. It is straightforward to extend our approach to multiple layers for a higher fidelity model, but a new multivariable method would need to be developed to search for the thicknesses and contrast values.

Around the $\mathrm{CH}_{4}$ absorption region $(\lambda \sim 890 \mathrm{~nm})$ in Fig. 13, the $\alpha_{\text {Jupiter }}$ curve dips significantly below $\alpha_{\text {flat }}$ and drops to zero, or slightly negative, for a few points. A close examination of the Jupiter frames in this region showed that the absorption feature is deep enough that the intensity signal-to-noise ratio is poor and the fringe variations are buried in the noise, which explains the $\alpha_{\text {Jupiter }}$ values dropping near zero. We found that applying an uncorrected flat-field introduced fringes back into these science frames. Thus, our approach of separate defringing of the flat-field frames is important for this wavelength region. The few negative points for $\alpha_{\text {Jupiter }}$ in this region might again be influenced by the multilayer response effect mentioned in the previous paragraph.

It is not clear if the optical spectral energy distribution differences for the flat-field lamps and Jupiter also contribute to the difference in $\alpha$-values near $\lambda \sim 890 \mathrm{~nm}$ because it is difficult to separate this from other effects. QTH lamps mounted to the APO 3.5-m telescope structure were used for flat-field illumination and APO test calibration results for these lamps show a relatively smooth spectral irradiance profile that increases with wavelength throughout our range of interest. On the other hand, the $\mathrm{CH}_{4}$ absorption feature involves large gradients so it is possible there is a spectral energy difference contribution to the $\alpha$-value results. We have yet to find a definitive explanation for the peak and dip variation in the $\alpha_{\text {flat }}$ curve for $\lambda>890 \mathrm{~nm}$; however, we saw a similar variation in other flat-field data collected for this CCD, so it may be device related.

\subsection{Disk-Averaged Spectrum with Defringed Jupiter Data}

The corrected NAIC Jupiter images undergo a geometric and a photometric calibration prior to any spectral analysis. Application of these data in conjunction with the radiative transfer package NEMESIS (nonlinear optimal estimator for multivariate spectral analysis) ${ }^{17}$ for modeling of the structure and color of Jupiter's uppermost cloud deck is ongoing. Nevertheless, as an initial verification of the defringing and calibration process, the disk-averaged NAIC Jupiter spectrum was compared with an established spectrum presented by Karkoschka. ${ }^{18}$ Figure 14 shows such

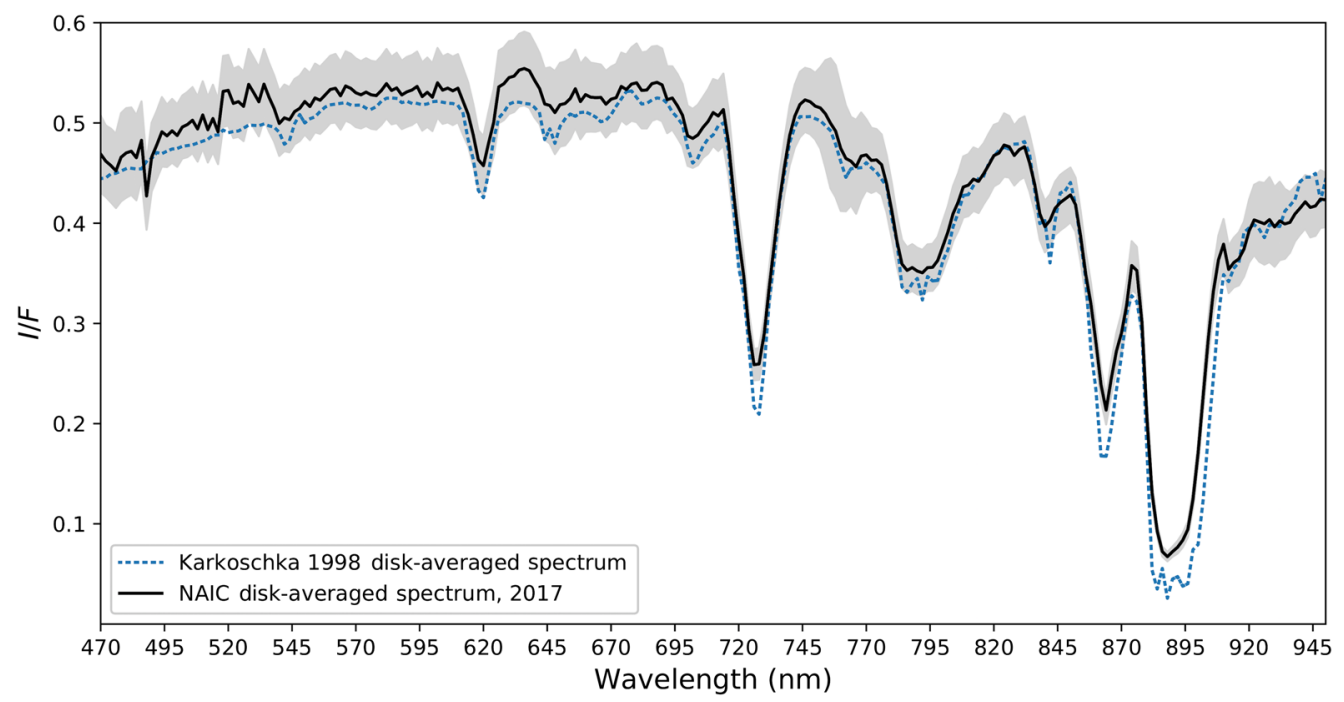

Fig. 14 Disk-averaged spectrum of NAIC Jupiter data that were defringed, calibrated, and corrected for spectral leakage compared to a spectrum from Karkoschka. ${ }^{18}$ The estimation error envelope for the NAIC spectrum is marked in gray. 
a comparison with defringed and calibrated NAIC data that were collected on March 27, 2017, during the 5th perijove pass of Juno. The Karkoschka spectrum falls within the error margins estimated for the NAIC spectrum, which suggests the defringing process did not significantly perturb the spectrum. Some slight differences between the spectra, particularly in the absorption bands, can be attributed to limited NAIC spectral resolution, low signal-to-noise ratio, and a sensitive spectral leakage correction that accounts for filter transmission sidelobes.

\section{Conclusions}

Based on wave optics field equations, an interference model was developed to correct etaloning fringes assuming an imaging sensor with a single layer. The model was used to derive the 2-D physical thickness function of the CCD sensor used in NAIC by analyzing a sequence of flatfield spectral images. The thickness function was found to have an overall dish-shaped variation along with some finely spaced surface polishing marks. The features of the function were consistent with the fringe characteristics seen in the flat-field images. Normalized synthetic fringe frames were created using the 2-D thickness function and correction of an image at a particular wavelength involved division by the appropriate synthetic intensity frame combined with a bestcorrection $\alpha$-value (related to fringe contrast) that is found with a search procedure to minimize the fringes in the image. The final science images were generated by dividing the fringecorrected Jupiter frames by the fringe-corrected flat-field frames.

After processing, the etaloning fringe contrast in the science images was reduced by a factor of 2 to 8 even for wavelength regions involving significant absorption features in Jupiter's optical spectrum. We also found that a poor signal-to-noise ratio in the science images in the absorption wavelength region caused the etaloning fringe variations to be obscured. However, the approach of separately defringing the flat-field and science images still allowed for good overall flat-field correction. The disk-averaged spectrum of the defringed and calibrated NAIC data compared favorably with an established spectrum for Jupiter, which further validates the defringing method.

We expect that this method is applicable to other detectors when a sequence of spectral images with adequate wavelength resolution is available and more beneficially, the method can be applied when the sensor structure is unknown.

\section{Acknowledgments}

This work was supported by the Research Support Agreement 1569980 from the Jet Propulsion Laboratory, as a subaward of NASA/Solar System Observation grant. The authors would like to thank Michael Wong at the University of California, Berkeley, for helpful discussions on etaloning and modeling the fringing, Hanyu Zhan and Robert Hull at New Mexico State University for their support of laboratory calibrations and installation of NAIC system at APO, and William Ketzeback at APO for providing information and insight on the QTH lamps within the APO 3.5-m telescope dome. The authors would also like to thank an anonymous reviewer who provided comments and suggestions that significantly improved this article.

\section{References}

1. E. M. Malumuth et al., "Removing the fringes from space telescope imaging spectrograph slitless spectra," Publ. Astron. Soc. Pac. 115(804), 218-234 (2003).

2. J. R. Walsh et al., "Modelling the fringing of the ACS WFC and HRC chips," ST-ECF Instrument Science Report ACS 2003-003 (2003).

3. S. B. Howell, "Fringe science: defringing CCD images with neon lamp flat fields," Publ. Astron. Soc. Pac. 124(913), 263-267 (2012).

4. M. H. Wong, "Amplitude of fringing in WFC3 /UVIS narrowband red filters," WFC3 Instrument Science Report 2010-04 (2010).

5. M. Kümmel et al., "The slitless spectroscopy data extraction software aXe," Publ. Astron. Soc. Pac. 121(875), 59-72 (2009). 
6. W. Ren et al., "Wavelet transform based defringing in interference imaging spectrometer," Opt. Express 25(15), 17039-17050 (2017).

7. E. M. Malumuth et al., "Model of fringing in the WFC3 CCDs," Proc. SPIE 4854, 567-576 (2003).

8. P. M. Rojo and J. Harrington, "A method to remove fringes from images using wavelets," Astrophys. J. 649(1), 553-560 (2006).

9. A. A. Simon, M. H. Wong, and G. S. Orton, "First results from the Hubble OPAL program: Jupiter in 2015," Astrophys. J. 812(1), 55 (2015).

10. E. Dahl et al., "Radiative transfer analysis of hyperspectral image cubes of Jupiter acquired during Juno's 5th perijove pass," in EPSC-DPS Jt. Meeting 2019, Vol. 13, pp. 1-2 (2019).

11. A. Adriani et al., "JIRAM, the Jovian Infrared Auroral Mapper," Space Sci. Rev. 213, 393 446 (2017).

12. M. A. Janssen et al., "MWR: microwave radiometer for the Juno mission to Jupiter," Space Sci. Rev. 213, 139-185 (2017).

13. S. J. Bolton et al., "Jupiter's interior and deep atmosphere: the initial pole-to-pole passes with the Juno spacecraft," Science 356, 821-825 (2017).

14. A. S. Braude et al., "Colour and tropospheric cloud structure of Jupiter from MUSE/VLT: retrieving a universal chromophore," Icarus 338, 113589 (2020).

15. E. Wijerathna et al., "Correction of etaloning effects in ground-based hyperspectral image cubes of Jupiter," in IEEE Aerosp. Conf. Proc., Big Sky, Montana (2019).

16. M. A. Green, "Self-consistent optical parameters of intrinsic silicon at $300 \mathrm{~K}$ including temperature coefficients," Sol. Energy Mater. Sol. Cells 92 (11), 1305-1310 (2008).

17. P. G. J. Irwin et al., "The NEMESIS planetary atmosphere radiative transfer and retrieval tool," J. Quantum Spectrosc. Radiat. Transf. 109(6), 1136-1150 (2008).

18. E. Karkoschka, "Methane, ammonia, and temperature measurements of the Jovian planets and Titan from CCD-spectrophotometry," Icarus 133(1), 134-146 (1998).

Erandi Wijerathna earned a BS (Honors) degree in physics from the University of Colombo, Sri Lanka, in 2011. She received her MS degree in physics and electrical engineering from New Mexico State University in 2016. Currently, she is a PhD student at the Klipsch School of Electrical and Computer Engineering, New Mexico State University. Her research interests include laser-beam propagation through atmospheric turbulence, image processing, and polarimetric lidar.

Emma Dahl received her BA degree in physics-astronomy from Whitman College in 2015. Currently, she is a PhD candidate at the New Mexico State University Astronomy Department, where she studies and observes Jupiter's atmosphere in support of NASA's Juno mission. Her other research interests include instrumentation and mission planning and design.

David Voelz earned a PhD in electrical engineering from the University of Illinois in 1987. He is a professor at the Klipsch School of Electrical and Computer Engineering, New Mexico State University. He has been involved in the development of optical systems involving AOTF components since 2002. His research interests include spectral and polarization sensing, laser-beam propagation through atmospheric turbulence, laser communications, imaging theory, and astronomical instrumentation development. He is a fellow of SPIE.

Nancy Chanover received her BA degree in physics from Wellesley College in 1991 and a PhD in astronomy from New Mexico State University in 1997. Currently, she is a professor of astronomy at New Mexico State University. Her current research activities include the development and advancement of innovative technologies for remote sensing and in situ applications in studies of solar system atmospheres and surfaces. 